Editorial

\title{
PSMA-PET for Lymph Node Detection in Recurrent Prostate Cancer: How do we use the Magic Bullet?
}

\author{
Tobias Maurer1, Declan G. Murphy², Michael S. Hofman², Matthias Eiber ${ }^{\natural}$ \\ 1. Department of Urology, Technical University of Munich, Munich, Germany \\ 2. Division of Cancer Surgery, Peter MacCallum Cancer Centre, Melbourne, Australia; Australian Prostate Cancer Research Centre, Epworth Healthcare, \\ Richmond, Australia; The Sir Peter MacCallum Department of Oncology, University of Melbourne \\ 3. Cancer Imaging, Peter MacCallum Cancer Centre, Melbourne, Australia; The Sir Peter MacCallum Department of Oncology, University of Melbourne \\ 4. Department of Nuclear Medicine, Technical University of Munich, Munich, Germany; Department of Molecular and Medical Pharmacology, David Geffen \\ School of Medicine, University of California Los Angeles, Los Angeles, CA, USA
}

$\square$ Corresponding author: PD Dr. med. Tobias Maurer, FEBU, Department of Urology, Technical University of Munich (TUM), Klinikum rechts der Isar, Ismaninger Str. 22, 81671 München. Tel.: 089-4140-2508; FAX: 089-4140-4843; e-mail: t.maurer@tum.de

(c) Ivyspring International Publisher. This is an open access article distributed under the terms of the Creative Commons Attribution (CC BY-NC) license (https://creativecommons.org/licenses/by-nc/4.0/). See http://ivyspring.com/terms for full terms and conditions.

Published: 2017.05.15

Currently, there is a surge in imaging in prostate cancer due to the recent advances in PET imaging using the prostate-specific membrane antigen as target structure. In their article, Jilg et al. [8] add significant knowledge on the true capability of this new imaging technique for the detection of lymph node metastases in patients with recurrent disease.

In the past, imaging in patients with biochemically recurrent prostate cancer did not play a major role. Especially at low prostate specific antigen (PSA) values, conventional imaging modalities like computed tomography (CT) or magnetic resonance imaging (MRI) or bone scintigraphy often cannot reliably detect and localize recurrent disease and therefore lack impact on treatment decisions. However, since the recent introduction of novel positron emission tomography (PET) tracers targeting the prostate-specific membrane antigen (PSMA), the role of imaging is changing rapidly [1]. These (almost) prostate cancer specific tracers are able to reveal sites of recurrence in a clarity clearly superior to still most commonly used agents like ${ }^{18} \mathrm{~F}$-fluoroethylcholine or ${ }^{11}$ C-choline [2].

Thus, increasing numbers of urologists, radiation physicians and oncologists perceive PSMA-based PET as the long-desired magic bullet to detect recurrent prostate cancer [3]. In turn this has led to a surge of imaging in these patients - despite the still limited experience with this novel technology. Furthermore, histological proof of detected lesions is only reported in a subset of publications and reported sensitivity and specificity rates differ significantly [4-7].

In the presented study, Jilg et al. provide additional data on the value (and limitation) of this new imaging technology in patients with biochemical recurrence. In this well-conducted analysis positive findings on ${ }^{68} \mathrm{Ga}-H B E D-C C-P S M A$ PET were validated by detailed histopathological assessment. [8]. Most importantly, it shows that a certain size of metastatic lesions within lymph nodes is needed $(50 \%$ and $90 \%$ detection rate at short axis diameters of $\geq 2.3$ $\mathrm{mm}$ and $\geq 4.5 \mathrm{~mm}$ ) to be picked up by ${ }^{68} \mathrm{Ga}-\mathrm{HBED}-\mathrm{CC}-\mathrm{PSMA}$ PET - reminding us that also this method is not perfect and fails to detect microscopic lesions mainly due to limited spatial resolution of PET as well as insufficient tracer accumulation. However, ${ }^{68} \mathrm{Ga}-\mathrm{HBED}-\mathrm{CC}-\mathrm{PSMA}$ PET detects metastatic lymph node lesions significantly earlier than conventional CT or MRI that usually requires a size of at least $8 \mathrm{~mm}$ [9]. Certainly, the true sensitivity of ${ }^{68} \mathrm{Ga}-\mathrm{HBED}-\mathrm{CC}-\mathrm{PSMA}$ PET in biochemical recurrence cannot be calculated from this study since only patients with positive findings were considered for salvage lymph node dissection (and that in part might explain the slightly different results to previously published studies in patients with primary prostate cancer where also PET-negative patients underwent surgery). On the contrary, this study also demonstrates the high accuracy for 
detection of lymph node metastases by this novel imaging technology. This is further underlined by the successful development of radioactively labeled PSMA-targeted molecules for intraoperative surgical guidance via gamma detectors [10-12].

But where do we proceed from here? Where is the manual on how to use the PSMA-targeted magic bullet? The perspicuity of ${ }^{68} \mathrm{Ga}-\mathrm{HBED}-\mathrm{CC}-\mathrm{PSMA}$ PET might suborn us to treat patients without considering the limitations of this evolving imaging (e.g. still missing microscopic disease). When offering patients currently considered experimental localized therapies like salvage lymph node dissection we should bear in mind the most important rule in medicine: "Primum nihil nocere, secundum cavere, tertium sanare".

Offering patients localized targeted strategies we have to be aware that we might miss sites of disease and that we impose certain morbidity on patients. We must always remember to treat patients and not their images, and in doing so need to account for the patients' clinical condition, the prostate cancer specific history and risk stratification as well as the outcome of standard conservative or observational treatments [13]. Careful patient selection, adequate and balanced counselling as well as thorough investigation of follow-up is mandatory. Here, prospective studies must be undertaken to provide the answers and the evidence needed for implementation into guidelines.

Once again, the authors have to be congratulated for their dedicated work-up of imaging as well as histological evaluation that in detail describes the diagnostic capability of ${ }^{68} \mathrm{Ga}-\mathrm{HBED}-\mathrm{CC}-\mathrm{PSMA} \mathrm{PET}$ as well as its limitations for lymph node staging. As PSMA-based PET imaging is currently increasingly utilized such critical evaluation with direct histological comparison is important.

\section{References}

[1] Maurer T, Eiber M, Schwaiger M, Gschwend JE. Current use of PSMA-PET in prostate cancer management. Nat Rev Urol. 2016;13:226-35.

[2] Perera M, Papa N, Christidis D, Wetherell D, Hofman MS, Murphy DG, et al. Sensitivity, Specificity, and Predictors of Positive 68Ga-Prostate-specific Membrane Antigen Positron Emission Tomography in Advanced Prostate Cancer: A Systematic Review and Meta-analysis. European urology. 2016;70:926-37.

[3] Mottaghy FM, Behrendt FF, Verburg FA. (68)Ga-PSMA-HBED-CC PET/CT: where molecular imaging has an edge over morphological imaging. Eur J Nucl Med Mol Imaging. 2016;43:394-6.

[4] Budaus L, Leyh-Bannurah SR, Salomon G, Michl U, Heinzer H, Huland H, et al. Initial Experience of (68)Ga-PSMA PET/CT Imaging in High-risk Prostate Cancer Patients Prior to Radical Prostatectomy. European urology. 2016;69:393-6.

[5] Maurer T, Gschwend JE, Rauscher I, Souvatzoglou M, Haller B, Weirich G, et al. Diagnostic Efficacy of (68)Gallium-PSMA Positron Emission Tomography Compared to Conventional Imaging for Lymph Node Staging of 130 Consecutive Patients with Intermediate to High Risk Prostate Cancer. The Journal of urology. 2016;195:1436-43.

[6] Rauscher I, Maurer T, Beer AJ, Graner FP, Haller B, Weirich G, et al. Value of 68Ga-PSMA HBED-CC PET for the Assessment of Lymph Node Metastases in Prostate Cancer Patients with Biochemical Recurrence: Comparison with Histopathology After Salvage Lymphadenectomy. Journal of nuclear medicine : official publication, Society of Nuclear Medicine. 2016;57:1713-9.
[7] van Leeuwen PJ, Emmett L, Ho B, Delprado W, Ting F, Nguyen Q, et al. Prospective evaluation of 68Gallium-prostate-specific membrane antigen positron emission tomography/computed tomography for preoperative lymph node staging in prostate cancer. BJU international. 2017;119:209-15.

[8] Jilg CA, Drendel V, Rischke HC, Beck T, Vach W, Schaal K, et al. Diagnostic accuracy of Ga-68-HBED-CC-PSMA-Ligand-PET/CT before salvage lymph node dissection for recurrent prostate cancer. Theranostics. 2017;7:1770-80.

[9] Hovels AM, Heesakkers RA, Adang EM, Jager GJ, Strum S, Hoogeveen YL, et al. The diagnostic accuracy of CT and MRI in the staging of pelvic lymph nodes in patients with prostate cancer: a meta-analysis. Clinical radiology. 2008;63:387-95.

[10] Maurer T, Weirich G, Schottelius M, Weineisen M, Frisch B, Okur A, et al. Prostate-specific membrane antigen-radioguided surgery for metastatic lymph nodes in prostate cancer. European urology. 2015;68:530-4.

[11] Rauscher I, Duwel C, Wirtz M, Schottelius M, Wester HJ, Schwamborn K, et al. Value of 111 In-prostate-specific membrane antigen (PSMA)-radioguided surgery for salvage lymphadenectomy in recurrent prostate cancer: correlation with histopathology and clinical follow-up. BJU international. 2016.

[12] Robu S, Schottelius M, Eiber M, Maurer T, Gschwend J, Schwaiger M, et al. Preclinical Evaluation and First Patient Application of 99mTc-PSMA-I\&S for SPECT Imaging and Radioguided Surgery in Prostate Cancer. Journal of nuclear medicine : official publication, Society of Nuclear Medicine. 2017;58:235-42.

[13] Murphy DG, Sweeny CJ, Tombal B. “Gotta catch 'em all”, or do we? Pokemet approach to metastatic prostate cancer. European urology. 2017; in press. 